Revue internationale P.M.E.

Économie et gestion de la petite et moyenne entreprise

\title{
Les effets autorenforçants de la coopération et des capacités d'innovation : une étude de PME françaises
}

\section{Frédéric Huet}

Volume 19, numéro 1, 2006

URI : https://id.erudit.org/iderudit/1008491ar

DOI : https://doi.org/10.7202/1008491ar

Aller au sommaire du numéro

Éditeur(s)

Presses de l’Université du Québec

ISSN

0776-5436 (imprimé)

1918-9699 (numérique)

Découvrir la revue

Citer cet article

Huet, F. (2006). Les effets autorenforçants de la coopération et des capacités d'innovation : une étude de PME françaises. Revue internationale P.M.E., 19(1), 95-117. https://doi.org/10.7202/1008491ar
Résumé de l'article

Cet article s'intéresse aux liens entre la coopération et l'innovation au sein des PME. La mobilisation d'une approche de l'organisation à dominante cognitive conduit à envisager ce lien de manière duale : l'innovation peut être considérée comme un résultat des interactions coopératives ou comme une condition de la coopération. Notre étude des PME, par la confrontation de données statistiques et d'études de cas, met en évidence certaines singularités dans les pratiques coopératives de ces petites organisations. Si les capacités d'absorption des firmes déterminent leur recours aux partenariats, l'impact de ceux-ci sur l'innovation reste plus variable : les synergies potentielles associées aux complémentarités des compétences semblent sous-exploitées et l'innovation émerge de manière nettement plus induite que délibérée et contrôlée. 


\title{
Les effets autorenforçants de la coopération et des capacités d'innovation: une étude de PME françaises
}

Frédéric HUET

Équipe COSTECH

Université de technologie de Compiègne

\section{MOTS CLÉS}

\author{
Coopération - Innovation - PME \\ Connaissances - Capacité d'absorption
}

\section{RÉSUMÉ}

Cet article s'intéresse aux liens entre la coopération et l'innovation au sein des PME. La mobilisation d'une approche de l'organisation à dominante cognitive conduit à envisager ce lien de manière duale: l'innovation peut être considérée comme un résultat des interactions coopératives ou comme une condition de la coopération. Notre étude des PME, par la confrontation de données statistiques et d'études de cas, met en évidence certaines singularités dans les pratiques coopératives de ces petites organisations. Si les capacités d'absorption des firmes déterminent leur recours aux partenariats, l'impact de ceux-ci sur l'innovation reste plus variable: les synergies potentielles associées aux complémentarités des compétences semblent sous-exploitées et l'innovation émerge de manière nettement plus induite que délibérée et contrôlée.

\section{L'AUTEUR}

FRÉDÉRIC HUET détient un doctorat en sciences économiques, spécialisé en économie de l'innovation et des organisations. Ses travaux sont focalisés sur le développement des relations coopératives dans les PME, les dispositifs de coordination qui peuvent y être associés et le rôle qu'elles peuvent jouer dans les dynamiques d'innovation. Il est membre du laboratoire Costech de l'Université de technologie de Compiègne et chargé d'études économiques au laboratoire LUTIN (UMS CNRS) dans le cadre d'un projet européen sur les mutations du secteur audiovisuel. Adresse: Équipe COSTECH (Connaissances, organisation et systèmes techniques), Département Technologie et sciences de l'Homme, Centre Pierre-Guillaumat, Université de technologie de Compiègne, B.P. 60649, 60206 Compiègne Cédex, France. Téléphone: 333442345 97, télécopieur: 333442352 12. Courriel <frederic.huet@utc.fr>.

(C) 2006 - Presses de l'Université du Québec

Édifice Le Delta I, 2875, boul. Laurier, bureau 450, Québec, Québec G1V 2M2 • Tél.: (418) 657-4399 - www.puq.ca

Tiré de: Revue internationale P.M.E., vol. 19, no 1, sous la direction de Louis Raymond • PME1901N

Tous droits de reproduction, de traduction et d'adaptation réservés 


\begin{abstract}
This article sheds light on the links between cooperation and innovation in SMEs. The investigation within a theoretical framework focused on knowledge leads to a dual link: innovation as a result of cooperative interactions or as a prerequisite to cooperation. Our study of SME's, by confronting statistical data and case studies, brings to the fore some singularities in this inter-organisational set-up. If the absorptive capacity of the firms strongly determine their resort to partnership, the impact of latter on innovation capacities remains more variable:potential synergies associated with the complementarity of competences seem under-achieved and the emergence of innovation is clearly more induced than deliberate and controlled.
\end{abstract}

\title{
RESUMEN
}

Este artículo se interesa a los vinculos entre la cooperación y la innovación en las PYMEs. La utilización de un enfoque de la organización predominantemente cognitiva, nos conduce a considerar este vínculo de manera dual: la innovación puede ser considerada como el resultado de las interacciones cooperativas o como un requisito a la cooperación. Nuestro análisis de las PYMEs, a través de la confrontación de datos estadísticos y de estudios de casos, pone en evidencia ciertas singularidades en las prácticas cooperativas de estas pequeñas organizaciones. Si las capacidades de absorción de las firmas determinan su recurso a asociados, el impacto de estos sobre la innovación permanece más variable: las potenciales sinergias aunadas a las competencias complementarias parecen sub-explotadas y la innovación surge de manera más inducida que deliberada y controlada.

\section{ZUSAMMENFASSUNG}

Dieser Artikel befasst sich mit den Verbindungen von Kooperationen und Innovationen innerhalb von KMU. Betrachtet man bei Organisationen ihre kognitive Dominanz, so muss die oben angesprochene Verbindung von zwei Standpunkten aus analysiert werden. Einerseits kann die Innovation als ein Ergebnis der gemeinsamen Wechselwirkungen angesehen werden. Andererseits ist die Innovation eine Voraussetzung zur Zusammenarbeit. Unsere Studie, gestützt auf der Gegenüberstellung von statistischen Werten und Fallstudien, hebt einige Besonderheiten der Zusammenarbeitsformen von kleinen und mittleren Unternehmen hervor. Ist die Aufnahmefähigkeit eines Unternehmens entscheidend, ob und in welcher Form Partnerschaften eingegangen werden, so ist die Auswirkung auf die Innovation stark variabel. Synergien aufgrund ergänzender Kompetenzen werden anscheinend ungenügend genutzt und Innovationen entstehen eher in abgeleiteter Form als kontrolliert und durch Beratung.

\section{Introduction}

L'objectif de cette contribution est d'éclairer le lien entre coopération et innovation. La coopération est souvent considérée comme une source potentielle d'innovation venant compléter les processus d'apprentissage interne des organisations. En

Revue internationale P.M.E., vol. 19, $\mathrm{n}^{\circ}$ 1, 2006

(C) 2006 - Presses de l'Université du Québec

Édifice Le Delta I, 2875, boul. Laurier, bureau 450, Québec, Québec G1V 2M2 • Tél.: (418) 657-4399 - www.puq.ca

Tiré de: Revue internationale P.M.E., vol. 19, n 1, sous la direction de Louis Raymond • PME1901N

Tous droits de reproduction, de traduction et d'adaptation réservés 
réunissant des partenaires aux bases de connaissances différentes, les partenariats stimulent le renouvellement des compétences propres de chaque firme. Souvent considérées comme moins innovantes en raison des plus faibles ressources qu'elles peuvent consacrer à l'innovation (Lhuillery, 1996), les PME pourraient trouver dans les formes coopératives un accès à l'innovation, difficile à générer en interne (Bougrain et Haudeville, 2002; Mustar, 2001; Bellotti, 2000). Cependant, cet apprentissage externe peut également être conditionné par leurs propres capacités internes. Dans ce cadre, les coopérations n' offrent des perspectives d'innovation que si les partenaires possèdent déjà leurs propres capacités d'apprentissage. Les capacités d'innovation, en plus d'être envisagées comme un résultat de la coopération, deviennent nécessaires à l'apprentissage externe.

Notre analyse se caractérise par sa dominante cognitive, dans laquelle l'organisation est conçue comme un ensemble cohérent de connaissances et de compétences et où l'innovation traduit la capacité d'apprentissage, de renouvellement et d'évolution de ses compétences. Le présent article se propose alors d'analyser les différents discriminants de l'innovation par la coopération (section 1).

L'analyse empirique, à travers une enquête réalisée auprès de PME françaises ${ }^{1}$, porte sur une base de données originale et sur des entretiens qualitatifs offrant des éléments pour mieux cerner les déterminants internes et externes de l'apprentissage coopératif. Différents blocs de variables ont ainsi été définis, pour apprécier les déterminants de l'apprentissage par la coopération: caractéristiques internes de la firme, configuration coopérative et impact de la coopération sur les capacités d'innovation (section 2).

L'ensemble de ces variables nous permet ainsi de relever les discriminants de l'engagement dans des relations coopératives (3.1), puis les caractéristiques internes (3.2) et les configurations coopératives (3.3) susceptibles de catalyser l'apprentissage coopératif.

\section{PME, coopération et innovation}

\subsection{L'innovation dans les théories basées sur la connaissance}

Dans les approches évolutionnistes des théories des organisations, la notion de connaissance occupe une place centrale dès lors qu'elle se constitue en élément différenciateur de chaque organisation. Ces ressources intangibles à la base des avantages compétitifs de la firme forgent son identité organisationnelle. La dimension cognitive est alors au centre des analyses organisationnelles.

1. Étude française dirigée par Lazaric et Rizopoulos, financée par l'État et la région Picardie (2003).

Revue internationale P.M.E., vol. 19, n 1, 2006

(C) 2006 - Presses de l'Université du Québec

Édifice Le Delta I, 2875, boul. Laurier, bureau 450, Québec, Québec G1V 2M2 • Tél.: (418) 657-4399 - www.puq.ca

Tiré de: Revue internationale P.M.E., vol. 19, no 1, sous la direction de Louis Raymond • PME1901N

Tous droits de reproduction, de traduction et d'adaptation réservés 
La connaissance ne peut se résumer à un seul traitement d'information. C'est plutôt au regard d'un manque d'informations que cette notion de connaissance prend son sens. Dès lors que l'information disponible sur l'environnement est incomplète et imparfaite, les prises de décision ne peuvent s'effectuer à partir des seuls calculs et traitements de l'information. Ces prises de décision reposent alors sur des schémas interprétatifs, issus des expériences passées et venant combler les «vides informationnels». Les connaissances traduisent ainsi la capacité des organisations à interpréter les informations imparfaites et incomplètes reçues de l'environnement. L'organisation, tout particulièrement la firme, est alors envisagée comme un processeur de connaissances, beaucoup plus que comme un processeur d'informations (Fransmann, 1994; Cohendet, 1998).

Ces connaissances organisationnelles, cristallisées dans des routines analogues aux gènes de la firme, se caractérisent par leur forte dimension tacite. Elles apparaissent difficilement codifiables et sont activées de manière quasi automatique par leurs détenteurs. Ainsi, l'accès aux connaissances et aux schémas interprétatifs qui les sous-tendent est rendu difficile par une explicitation qui ne peut être que partielle (Neslon et Winter, 1982). Cette dimension tacite engendre alors une certaine inertie organisationnelle, une conformité aux habitudes et institutions qui tend à reconduire les pratiques et schémas cognitifs à l'œuvre (Hodgson, 1988).

L'innovation traduit, dans ce cadre, le changement de cette base de connaissances, le renouvellement de son répertoire en réponse à des modifications environnementales rendant partiellement obsolètes les connaissances disponibles. Par rapport à la dimension tacite des connaissances, le changement est le fruit de processus d'apprentissage, résultant de stratégies exploratoires venant réviser et modifier les répertoires de connaissances en place. La capacité des organisations à innover est alors assimilable aux capacités d'apprentissage des organisations (March, 1991).

\subsection{Taille de la firme et capacités d'innovation}

L'influence de la taille de la firme sur sa capacité à introduire du changement reste relativement ambiguë et controversée dans la littérature organisationnelle, à l'image du paradoxe schumpétérien, illustrant la difficulté à rendre compte de cette relation entre la taille et la capacité d'innovation (Munier, 2004; Huet, 2004).

La première période des travaux de Schumpeter (Schumpeter I) met en avant la plus grande capacité des petites organisations à engendrer de l'innovation. En effet, la flexibilité de ces petites structures permet une concrétisation plus aisée des stratégies entrepreneuriales, dès lors que le poids de l'organisation bureaucratique se fait moins sentir. Les petites entreprises apparaissent alors plus aptes à enclencher les processus de «destruction créatrice». À l'inverse, une inertie organisationnelle se

Revue internationale P.M.E., vol. 19, $n^{\circ} 1,2006$

(C) 2006 - Presses de l'Université du Québec

Édifice Le Delta I, 2875, boul. Laurier, bureau 450, Québec, Québec G1V 2M2 • Tél.: (418) 657-4399 - www.puq.ca

Tiré de: Revue internationale P.M.E., vol. 19, $\mathrm{n}^{\circ}$ 1, sous la direction de Louis Raymond • PME1901N

Tous droits de reproduction, de traduction et d'adaptation réservés 
développe dans les plus grandes firmes. Les rigidités qui y sont associées viennent alors altérer les capacités de changement et d'adaptation des grandes organisations (Hannan et Freeman, 1977).

En effet, la primauté des règles et l'établissement de comportements procéduriers conduisent l'organisation à une certaine rigidité, étouffant les initiatives susceptibles d'introduire le changement nécessaire à son évolution. L'intériorisation de ces règles par l'ensemble des membres de l'organisation leur confère une valeur intrinsèque indépendante des objectifs. Ainsi, la taille croissante de la firme affecte négativement ses capacités d'innovation (Schumacher, 1978; March et Simon, 1958). Cette inertie est donc d'origine institutionnelle, étant donné qu'elle se réfère à l'ensemble des mécanismes de décision ou schémas cognitifs sous-tendant les activités organisationnelles.

Cependant, et le glissement de la vision de Schumpeter (Schumpeter II) reflète cet aspect de l'innovation, le processus de changement ne repose pas uniquement sur des stratégies entrepreneuriales individuelles, mais résulte de travaux de laboratoires de recherche et concerne de multiples acteurs. La remise en cause de cette «vision mythique» de l'entrepreneur vient alors tempérer cette supériorité des petites entreprises à innover. Autrement dit, la prise en compte du coût important associé au processus innovant favorise les plus grosses structures, disposant de plus de ressources pour financer les opérations de recherche et développement, à la base des processus d'apprentissage. Une seconde source d'inertie, provisionnelle, en référence aux ressources mobilisables et nécessaires pour l'engagement dans des activités d'innovation, semble donc affecter plus fortement les petites firmes que les grandes, bénéficiant, d'une part, de plus grandes variété et facilité de moyens de financement et, d'autre part, d'une moindre vulnérabilité face aux risques inhérents à tout projet innovant (Audretsch, 1994).

Par ailleurs, les petites structures reposent plus sur des compétences tacites que les grandes. En effet, les processus de supervision directe et la division du travail moins importante autorisent une coordination nettement moins explicite entre les membres de l'organisation que dans des structures plus imposantes et plus complexes. Le répertoire de connaissances des petites organisations se révèle alors nettement plus tacite et donc, soumis à une plus forte inertie freinant les processus d'apprentissage et de changement (Nooteboom, 2000). En effet, l'activation non délibérée et automatique des connaissances tacites offre un certain «confort intellectuel» et autorise une économie des ressources cognitives qui conduisent à certaines réticences au changement, ce dernier reposant sur une délibération plus importante. À l'inverse, la nature plus codifiée des connaissances de la grande firme permet une meilleure identification et articulation des connaissances pour venir les modifier (Hodgson, 1988; Robertson et Langlois, 1994).

Revue internationale P.M.E., vol. 19, n 1, 2006

(C) 2006 - Presses de l'Université du Québec

Édifice Le Delta I, 2875, boul. Laurier, bureau 450, Québec, Québec G1V 2M2 • Tél.: (418) 657-4399 - www.puq.ca

Tiré de: Revue internationale P.M.E., vol. 19, n 1, sous la direction de Louis Raymond • PME1901N

Tous droits de reproduction, de traduction et d'adaptation réservés 
À l'image des arguments théoriques qui peuvent renforcer les deux points de vue sur la relation entre la taille de l'organisation et la capacité d'innovation, les études empiriques ne permettent pas de venir conforter l'un ou l'autre des effets de taille supposés, venant plutôt alimenter chacune des visions selon les propositions (Nooteboom, 1999).

\subsection{Coopération et innovation}

Une des limites des petites entreprises mise en avant pour soutenir l'hypothèse de leur moindre potentiel apprenant est le peu de ressources disponibles pour piloter les processus d'innovation. Les PME ont ainsi des capacités beaucoup moins importantes que les grandes entreprises à s'engager dans des processus d'apprentissage autonomes et internes. Différents travaux, basés sur l'enquête européenne CIS, montrent ainsi le moindre dynamisme des PME par rapport aux grandes firmes (Miotti et Sachwald, 2003; Kremp et Mairesse, 2002; Tether, 2002).

Devant cette difficulté, les processus d'apprentissage externe peuvent se substituer aux processus internes de R-D. La coopération, en tant que mode d'interaction privilégié au-delà des seuls mécanismes de marché entre deux ou plusieurs organisations, offre alors des occasions d'apprentissage au fil des opérations conjointement réalisées par les partenaires (Lazaric et Marengo, 2000; Helper, MacDuffie et Sabel, 2000; Lundvall, 1988).

La coopération se définit alors comme une association entre plusieurs entreprises indépendantes qui choisissent de réaliser un projet ou une activité donnés en coordonnant les compétences, moyens et ressources nécessaires pour le mener à terme (Garette et Dussauge, 1995). Cette définition englobante souligne l'entente explicite entre les partenaires, autour d'au moins un objectif partagé, pour le développement d'activités à vocation commerciale et/ou technologique, sous des formes pouvant aller de relations renforcées de sous-traitance à la création d'entités juridiques telles les coentreprises ou joint-ventures (Nooteboom, 1999). La création de ces formes coopératives (qu'elles soient horizontales ou verticales) met en évidence le développement d'activités productives au-delà de la firme. Ces activités s'ancrent dans des réseaux ou faisceaux de liens interorganisationnels qui remettent en cause la dualité firme/marché fréquemment envisagée en théorie des organisations (Voisin, 2000; Gulati, 1998).

Ainsi, en réunissant des organisations possédant leur propre base de connaissances, les coopérations permettent un renouvellement des compétences organisationnelles, grâce aux interactions organisées de manière à permettre cette appropriation. Autrement dit, les «ponts» créés entre les différentes visions organisationnelles favorisent les processus d'apprentissage et le développement des capacités d'innovation de chaque partenaire. La coopération apparaît alors comme

Revue internationale P.M.E., vol. 19, $\mathrm{n}^{\circ} 1,2006$

(C) 2006 - Presses de l'Université du Québec

Édifice Le Delta I, 2875, boul. Laurier, bureau 450, Québec, Québec G1V 2M2 • Tél.: (418) 657-4399 - www.puq.ca

Tiré de: Revue internationale P.M.E., vol. 19, no 1, sous la direction de Louis Raymond PME1901N

Tous droits de reproduction, de traduction et d'adaptation réservés 
une voie privilégiée d'accès à l'innovation (Alm et McKelvey, 1999). Ces occasions d'apprentissage externe peuvent ainsi venir pallier les déficits internes des petites entreprises.

\subsection{Capacité d'absorption et coopération}

La capacité des entreprises à identifier et à intégrer les connaissances externes repose également sur les caractéristiques propres de la firme, au-delà de la configuration coopérative. La capacité d'absorption des firmes traduit les capacités organisationnelles à identifier, assimiler et exploiter les informations reçues de l'environnement. C'est cette capacité d'absorption qui détermine l'engagement et la conduite de processus d'apprentissage externe (Cohen et Levinthal, 1990).

Ainsi, les modèles de capacité d'absorption soulignent l'importance des capacités propres des firmes à développer un apprentissage externe. Cette capacité d'absorption repose en partie sur les capacités de R-D interne des firmes. Si les structures et activités de R-D se concrétisent par l'introduction d'innovations tangibles, elles développent également leurs capacités d'apprentissage, leur permettant d'apprendre à apprendre (Cohen et Levinthal, 1989). Les firmes les plus dynamiques et les plus engagées dans des processus d'innovation ont alors une meilleure capacité à développer des processus d'apprentissage externe lors de processus coopératifs. Ce lien entre capacité d'absorption et apprentissage coopératif induit alors un effet cumulatif susceptible d'avoir des conséquences structurelles entre des firmes pouvant accentuer leur dynamisme par le levier coopératif et d'autres «s'enfermant» dans un statisme que la coopération ne peut pallier.

Pour résumer les deux versants de ces travaux sur l'apprentissage externe, il apparaît que les coopérations offrent, par leur configuration, un potentiel pour les partenaires d'augmenter leurs capacités d'innovation. Cependant, l'effectivité de cette innovation repose en partie sur les capacités d'apprentissage que les organisations ont su développer et accumuler préalablement. Ainsi, la question qui guide l'étude que nous avons pu réaliser et que nous allons maintenant présenter est la suivante: le dynamisme organisationnel et l'innovation doivent-ils être envisagés comme le résultat d'activités coopératives ou comme leur condition?

\section{Présentation de l'étude}

\subsection{Les différents échantillons de l'étude}

Notre étude se compose de trois échantillons «gigognes» correspondant aux différentes données que nous avons pu recueillir sur les firmes de notre échantillon global. Le schéma 1 indique l'articulation entre ces différents échantillons. L'ensemble de l'échantillon concerne des données relatives à des entreprises de

Revue internationale P.M.E., vol. 19, nº 1, 2006

(C) 2006 - Presses de l'Université du Québec

Édifice Le Delta I, 2875, boul. Laurier, bureau 450, Québec, Québec G1V 2M2 • Tél.: (418) 657-4399 - www.puq.ca

Tiré de: Revue internationale P.M.E., vol. 19, n 1, sous la direction de Louis Raymond • PME1901N

Tous droits de reproduction, de traduction et d'adaptation réservés 
10 à 500 salariés, respectant ainsi la dénomination usuelle des PME, dans quatre secteurs distincts de l'industrie et des services, d'intensité technologique diverse: agroalimentaire, travail des métaux, composants électroniques et sociétés de service en ingénierie informatique.

TABLEAU 1

Caractéristiques sectorielles de l'étude

\begin{tabular}{lccc}
\hline Secteurs & $\begin{array}{c}\text { Intensité } \\
\text { technologique }\end{array}$ & $\begin{array}{c}\text { Nombre } \\
\text { de firmes }\end{array}$ & $\begin{array}{c}\text { Effectif } \\
\text { salarié moyen }\end{array}$ \\
\hline Agroalimentaire & Faible & 192 & 66 \\
\hline Travail des métaux & Faible & 249 & 41 \\
\hline Composants électroniques & Moyenne-forte & 109 & 91 \\
\hline SS2I & Forte & 88 & 57 \\
\hline Total & & 638 & 59 \\
\hline
\end{tabular}

Figure 1

Présentation des différents échantillons

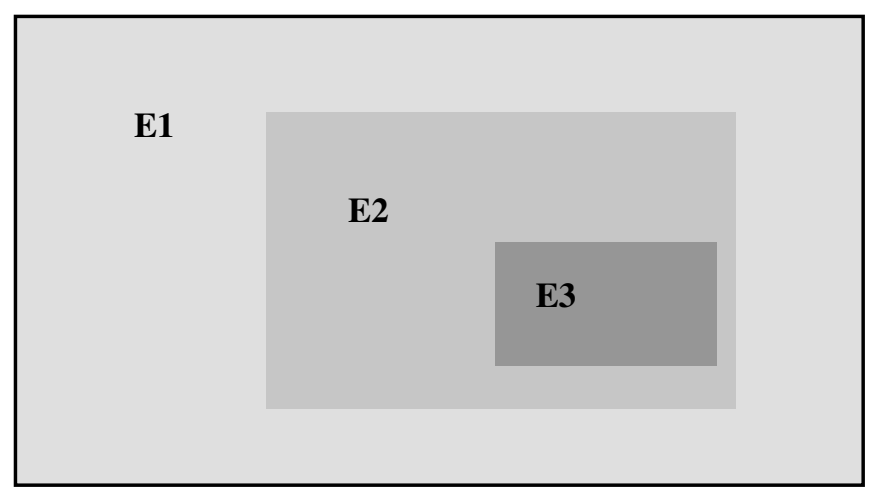

La première phase de l'étude est relative à la constitution d'une base de données statistiques de PME françaises. Le recueil des données a été effectué à partir d'un questionnaire administré par voie téléphonique. L'échantillon E1 comporte ainsi des données sur 638 PME françaises: ces données concernent les caractéristiques générales des firmes questionnées.

L'échantillon E2 réunit l'ensemble des PME qui, à l'intérieur de cet échantillon E1, ont déclaré développer des relations coopératives (de différentes natures: client-fournisseur, entre concurrents, GIE, groupement d'achat...), au-delà des seules relations de marché. Cet échantillon de 97 entreprises réunit des informations 
sur la coopération considérée la plus importante par les interviewés: caractéristiques de la relation et du partenaire, évolution de la relation, impact de la coopération sur les activités de l'entreprise, flux d'informations entre les partenaires.

L'échantillon E3 est relatif à la seconde partie de l'étude, à caractère qualitatif. À l'issue du recueil de données statistiques mentionné, des entreprises ont été sélectionnées à partir de l'échantillon E2 afin de réaliser des entretiens auprès d'acteurs de la coopération pour approfondir les informations extraites de la base de données. Vingt-deux entretiens ont ainsi été réalisés et retranscrits. Ces entretiens avaient pour objectif de comprendre plus finement, à travers des exemples concrets, les pratiques coopératives des PME, et ainsi fournir une base de discussion enrichissant les résultats statistiques.

\subsection{Définition d'indicateurs et tests réalisés}

Des indicateurs composites permettant de connaître les caractéristiques des firmes et des coopérations ont été construits à partir des différents items présents dans le questionnaire. La terminologie utilisée et la spécification des indicateurs sont synthétisés dans le tableau 2. Trois grands groupes d'indicateurs structurent ainsi l'étude.

- Les indicateurs relatifs aux caractéristiques internes de l'organisation: taille, âge, capacité d'absorption (par l'identification d'activités de R-D ou d'innovations récentes), rapport à la coopération.

- Les indicateurs relatifs à la configuration coopérative, pour apprécier et qualifier plus précisément cette coopération, par rapport aux échanges partenariaux (informations, degré d'interactions, durée de la relation), aux contenu et objectif des activités conjointes et au positionnement relatif des partenaires (taille, complémentarités des compétences, relation verticale ou horizontale).

- Les indicateurs relatifs à l'impact de la coopération sur les connaissances et compétences des PME. Ces impacts ont été mesurés sur le développement des compétences technologiques, l'amélioration de la qualité et l'évolution des pratiques de travail, à partir d'échelles d'évaluation de 1 à 5 .

Revue internationale P.M.E., vol. 19, nº 1, 2006

(C) 2006 - Presses de l'Université du Québec

Édifice Le Delta I, 2875, boul. Laurier, bureau 450, Québec, Québec G1V 2M2 • Tél.: (418) 657-4399 - www.puq.ca

Tiré de: Revue internationale P.M.E., vol. 19, $\mathrm{n}^{\circ} 1$, sous la direction de Louis Raymond • PME1901N

Tous droits de reproduction, de traduction et d'adaptation réservés 
TABLEAU 2

Indicateurs définis à partir du questionnaire

\begin{tabular}{|c|c|c|}
\hline & Indicateurs & Variables \\
\hline \multirow{7}{*}{ 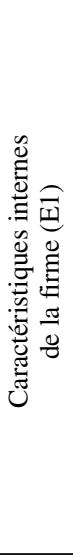 } & Âge & $\begin{array}{l}\text { Moins de } 5 \text { ans } \\
\text { Plus de } 5 \text { ans }\end{array}$ \\
\hline & Taille & $\begin{array}{l}\text { Moins de } 50 \text { salariés } \\
\text { Plus de } 50 \text { salariés }\end{array}$ \\
\hline & $\begin{array}{l}\text { R-D - Capacité } \\
\text { d'absorption }\end{array}$ & $\begin{array}{l}\text { Activités internes de R-D } \\
\text { Pas de R-D interne }\end{array}$ \\
\hline & Flexibilité interne & $\begin{array}{l}\text { Réorganisations récentes } \\
\text { Pas de réorganisation récente }\end{array}$ \\
\hline & Innovation interne & $\begin{array}{l}\text { Entreprise innovante (produits, procédés) } \\
\text { Entreprise non innovante }\end{array}$ \\
\hline & Coopération & $\begin{array}{l}\text { Liens interfirmes présents } \\
\text { Pas de liens interfirmes }\end{array}$ \\
\hline & Attitude coopérative & $\begin{array}{l}\text { Proactive (firme volontariste et en recherche de partenaires) } \\
\text { Réactive (coopération non initiée par la firme) }\end{array}$ \\
\hline \multirow{8}{*}{ 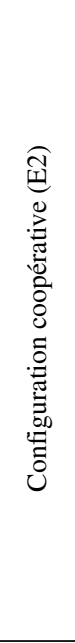 } & Nature & $\begin{array}{l}\text { Coopération verticale } \\
\text { Coopération horizontale }\end{array}$ \\
\hline & Durée de la relation & $\begin{array}{l}\text { Récente (moins de deux ans) } \\
\text { Long terme (plus de deux ans) }\end{array}$ \\
\hline & Objectifs d'innovation & $\begin{array}{l}\text { Objectifs initiaux d'innovation } \\
\text { Pas d'objectifs initiaux d'innovation }\end{array}$ \\
\hline & Contenu de la relation & $\begin{array}{l}\text { Coopération technologique } \\
\text { Coopération commerciale }\end{array}$ \\
\hline & Complémentarité & $\begin{array}{l}\text { Compétences complémentaires } \\
\text { Compétences similaires }\end{array}$ \\
\hline & Interactions & $\begin{array}{l}\text { Interactions fortes (équipes communes) } \\
\text { Interactions modérées (pas d'équipe commune) }\end{array}$ \\
\hline & Taille relative & $\begin{array}{l}\text { Différente } \\
\text { Similaire }\end{array}$ \\
\hline & $\begin{array}{l}\text { Échange } \\
\text { d'informations }\end{array}$ & $\begin{array}{l}\text { Renforcé } \\
\text { Modéré }\end{array}$ \\
\hline \multirow{3}{*}{ 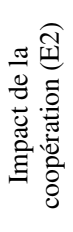 } & $\begin{array}{l}\text { Compétences } \\
\text { technologiques }\end{array}$ & \multirow{3}{*}{ Évaluation sur des échelles de Likert (1 à 5) } \\
\hline & $\begin{array}{l}\text { Amélioration } \\
\text { de la qualité }\end{array}$ & \\
\hline & $\begin{array}{l}\text { Évolution des pratiques } \\
\text { de travail }\end{array}$ & \\
\hline
\end{tabular}

Revue internationale P.M.E., vol. 19, $\mathrm{n}^{\circ} 1,2006$

(C) 2006 - Presses de l'Université du Québec

Édifice Le Delta I, 2875, boul. Laurier, bureau 450, Québec, Québec G1V 2M2 • Tél.: (418) 657-4399 - www.puq.ca

Tiré de: Revue internationale P.M.E., vol. 19, $\mathrm{n}^{\circ}$ 1, sous la direction de Louis Raymond • PME1901N

Tous droits de reproduction, de traduction et d'adaptation réservés 
Des tests de corrélation ont été appliqués à nos échantillons. Ils diffèrent selon la nature des données analysées: test de Khi²-Pearson pour l'analyse des données qualitatives (discriminants de l'engagement dans des activités coopératives) et test de Mann-Whithney pour l'analyse des données quantitatives (discriminants de l'impact de la coopération). Ces tests ont permis d'analyser:

- les caractéristiques organisationnelles favorisant le développement d'activités coopératives: comparaison, au sein de E1, des caractéristiques des firmes coopérant et ne coopérant pas. Ces analyses permettent ainsi de relever les variables discriminantes par rapport à l'aptitude à coopérer des PME (résultats en 3.1);

- les discriminants catalysant l'innovation par la coopération (une fois cette dernière engagée), à partir des corrélations établies (pour l'échantillon E2) entre les caractéristiques organisationnelles et l'impact mesuré de la coopération. Ces caractéristiques organisationnelles sont à la fois propres à la PME (résultats 4-2) et relatifs à la configuration coopérative (résultats 4-3).

Les entretiens seront mobilisés afin d'illustrer ou émettre des hypothèses par rapport à la signification des résultats statistiques présentés dans la partie suivante. Leur comparaison avec des statistiques parfois contre-intuitives permet ainsi de développer des pistes d'interprétation de ces résultats.

\section{Résultats de l'étude - discussion}

\subsection{Les $\mathrm{PME}$ : une faible aptitude à coopérer}

Le taux de coopération observé dans notre échantillon reste relativement faible: sur 638 firmes questionnées (E1), seules 97 ont déclaré être engagées dans des relations coopératives (E2), soit $15 \%$ de l'échantillon total. Ce résultat ne peut être considéré comme représentatif du taux de coopération des PME françaises; les biais associés à un recueil de données par voie téléphonique réduisent le taux de coopération réel de ces PME. Cependant, ce résultat met en évidence la faible propension à coopérer des PME, notamment par rapport aux grandes entreprises. Ce faible taux de coopération a déjà été souligné: alors que les stratégies coopératives sont courantes dans les grandes firmes, elles apparaissent beaucoup plus marginales dans les PME (Bayona, Garcia-Marco et Huerta, 2001).

Lors des interviews, cette faible capacité par les dirigeants de PME à envisager les avantages associés aux relations partenariales a souvent été abordée par l'évocation de mentalités individualistes et par la difficulté à concevoir des associations entre firmes potentiellement concurrentes. Ainsi, ce dirigeant de bureau d'étude dans le secteur de la mécanique illustre bon nombre de propos récurrents

Revue internationale P.M.E., vol. 19, nº 1, 2006

(C) 2006 - Presses de l'Université du Québec

Édifice Le Delta I, 2875, boul. Laurier, bureau 450, Québec, Québec G1V 2M2 • Tél.: (418) 657-4399 - www.puq.ca

Tiré de: Revue internationale P.M.E., vol. 19, n 1, sous la direction de Louis Raymond • PME1901N

Tous droits de reproduction, de traduction et d'adaptation réservés 
lors des interviews: "C'est dur à bouger: bouger les mentalités et pas que des personnes. C'est vrai qu'il y a un gros cap à passer à changer les mentalités. Donc, bouger les mentalités, c'est ce qu'il y a de plus dur.»

Malgré ces réticences à la coopération, certaines PME s'engagent dans des partenariats et remettent en cause les stratégies «autarciques» de développement et de «porte close». Les compétences internes des firmes permettent de rendre compte de ces variables discriminantes à l'égard de l'engagement coopératif.

\subsection{Les déterminants internes de l'apprentissage coopératif}

La comparaison entre les firmes déclarant nouer des relations coopératives et celles déclarant ne pas en nouer offrent des différences significatives par rapport aux critères de différenciation suivants: la taille de la firme, la présence d'activités de $\mathrm{R}-\mathrm{D}$, la réorganisation récente des activités et l'introduction récente d'innovations. Ces résultats sont présentés dans les tableaux 3 et 4.

Ces résultats mettent en évidence le rôle de la capacité d'absorption dans le développement de formes coopératives: les firmes possédant une telle capacité sont nettement plus engagées dans des coopérations que celles n'en bénéficiant pas. Mesurée au regard des intrants de l'innovation par les activités de R-D et par les résultats en termes de réorganisation et d'innovation, cette capacité d'absorption paraît déterminante pour la disposition des firmes à établir des partenariats. Notons que cette comparaison ne révèle qu'une différence significative sur l'engagement dans des pratiques coopératives et pas dans les résultats des processus d'apprentissage coopératif.

À partir des entretiens que nous avons pu mener, deux interprétations complémentaires permettent d'expliciter ce lien entre dynamisme interne et aptitude à coopérer. Tout d'abord, les firmes dotées de cette capacité d'absorption semblent les plus aptes à détecter les opportunités partenariales et les avantages potentiels de ces modes d'organisation. Cette capacité à envisager les «bienfaits» de la coopération résulte d'une stratégie entrepreneuriale de développement par l'innovation qui conduit à développer des capacités internes et externes d'apprentissage. Par ailleurs, la présence de ce dynamisme interne fait figure d'avantage concernant l'attractivité de la firme pour des partenaires potentiels. En effet, les compétences spécifiques et les capacités d'innovation de ces firmes permettent une meilleure identification de leur «valeur ajoutée» dans un cadre partenarial. Ce dynamisme interne joue alors un double rôle par rapport aux aptitudes coopératives. Il se révèle être un facteur d'identification à la fois de partenariats potentiels mais aussi pour les partenaires potentiels. Autrement dit, ce dynamisme favorise l'appréciation de l'environnement par la firme et de la firme par son environnement.

Revue internationale P.M.E., vol. 19, $n^{\circ} 1,2006$

(C) 2006 - Presses de l'Université du Québec

Édifice Le Delta I, 2875, boul. Laurier, bureau 450, Québec, Québec G1V 2M2 • Tél.: (418) 657-4399 - www.puq.ca

Tiré de: Revue internationale P.M.E., vol. 19, $\mathrm{n}^{\circ}$ 1, sous la direction de Louis Raymond - PME1901N

Tous droits de reproduction, de traduction et d'adaptation réservés 
TABLEAU 3

Distribution des firmes selon leurs caractéristiques organisationnelles (échantillon E1)

\begin{tabular}{lcccc}
\hline Indicateurs & Variable & $\begin{array}{c}\text { Pas de relations } \\
\text { coopératives } \\
(\mathbf{\%})\end{array}$ & $\begin{array}{c}\text { Relations } \\
\text { coopératives } \\
(\mathbf{\%})\end{array}$ & $\begin{array}{c}\text { Total } \\
(\mathbf{\%})\end{array}$ \\
\hline \multirow{2}{*}{ R-D } & Pas de R-D interne & 71 & 34 & 65 \\
\hline \multirow{2}{*}{ Innovation } & R-D interne & 29 & 66 & 35 \\
\hline \multirow{2}{*}{ Flexibilité } & Pas d'innovation récente & 68 & 47 & 65 \\
& Innovation récente & 32 & 53 & 35 \\
\hline \multirow{2}{*}{ Taille } & Modifications récentes & 16 & 65 & 81 \\
& Petite entreprise & 71 & 35 & 19 \\
\hline \multirow{2}{*}{ Âge } & Moyenne entreprise & 29 & 58 & 69 \\
& Jeune entreprise & 5 & 42 & 31 \\
\hline
\end{tabular}

TABLEAU 4

Corrélations entre les caractéristiques internes des firmes et la présence de relations coopératives (échantillon E1)

\begin{tabular}{lccc}
\hline Indicateurs & Sous-échantillons comparés & $\begin{array}{c}\text { Corrélation } \\
\text { par rapport } \\
\text { aux relations } \\
\text { coopératives }\end{array}$ \\
\hline R-D & R-D interne & Pas de R-D interne & $\begin{array}{c}50,035 \\
\mathrm{p}=0,000^{*}\end{array}$ \\
\hline Innovation & Récente & Non récente & $\begin{array}{c}15,369 \\
\mathrm{p}\end{array}$ \\
\hline Flexibilité & Modification récente & modification récente & $\begin{array}{c}17,284 \\
\mathrm{p}=0,000^{*}\end{array}$ \\
\hline Taille & $<50$ salariés & $>50$ salariés & $\begin{array}{c}7,189 \\
\mathrm{p}\end{array}$ \\
\hline Âge & Moins de cinq ans & Plus de cinq ans & $\begin{array}{c}\text { Pas de } \\
1,904\end{array}$ \\
\hline
\end{tabular}

Test de corrélation de Khi²-Pearson

* Différence significative au seuil de 0,01. 
L'impact des caractéristiques de la firme sur les résultats coopératifs fait également apparaître des différences significatives (voir tableau 5). Nos résultats montrent que les firmes possédant leurs propres activités de R-D tirent plus profit des opportunités technologiques au sein de la coopération. De même, les entreprises innovantes semblent plus aptes à faire évoluer leurs propres pratiques de travail après avoir vécu une expérience d'apprentissage coopératif. Enfin, dans le cadre de ces caractéristiques de la firme, l'attitude «proactive» des entreprises à l'égard de la coopération semble aussi déterminante dans les résultats de l'apprentissage. Les firmes engagées de manière volontaire et en demande de partenariats tirent nettement plus profit de la coopération que les firmes engagées à la suite des incitations institutionnelles ou de fortes contraintes environnementales. La recherche active d'opportunités partenariales, traduisant ainsi les bienfaits considérés de la coopération par ses initiateurs, place alors les partenaires dans des dispositions favorables et réceptives à l'apprentissage. Ces résultats soulignent l'influence du dynamisme interne pour tirer profit de l'expérience coopérative. Autrement dit, au-delà du déterminisme des compétences propres de la firme (capacité d'absorption), l'apprentissage n'apparaît pas comme un processus spontané, mais intentionnel et sous-tendu par des visions entrepreneuriales qui stimulent leur déclenchement.

Un producteur de flacons de parfums et de médicaments, ancien cadre dans une grande entreprise et désormais dirigeant de PME, relève la difficulté à développer les connaissances et les échanges dans une petite entreprise. Les relations partenariales représentent alors une source importante de renouvellement des connaissances: «Vous avez moins de temps, et ça c'est vrai, pour l'avoir constaté, dans une petite entreprise que dans une grande. Et le seul moyen que vous avez d'avoir des connaissances ou des échanges, c'est d'avoir des relations avec d'autres, que vous alliez les chercher. Aller les chercher, vous pouvez aller les chercher auprès de vos clients.»

Ainsi, la capacité d'absorption des firmes n'est pas neutre dans la dynamique coopérative. Elle influence tout d'abord fortement le développement des coopérations au sein des PME. En amont des processus d'apprentissage coopératif, cette capacité se traduit en outre par une propension accrue de détection des opportunités d'apprentissage pour les firmes. Ensuite, elle facilite l'apprentissage externe, par le renforcement des capacités d'innovations technologiques et organisationnelles. Ces résultats révèlent l'existence d'une dynamique d'autorenforcement dans les capacités d'innovation des PME: les firmes disposant initialement de capacités d'apprentissage semblent pouvoir activer et bénéficier d'un levier coopératif pour accroître ces compétences, alors qu'à l'inverse, les firmes ne disposant pas de telles compétences ne semblent pas pouvoir développer des stratégies coopératives pour pallier ce manque. 


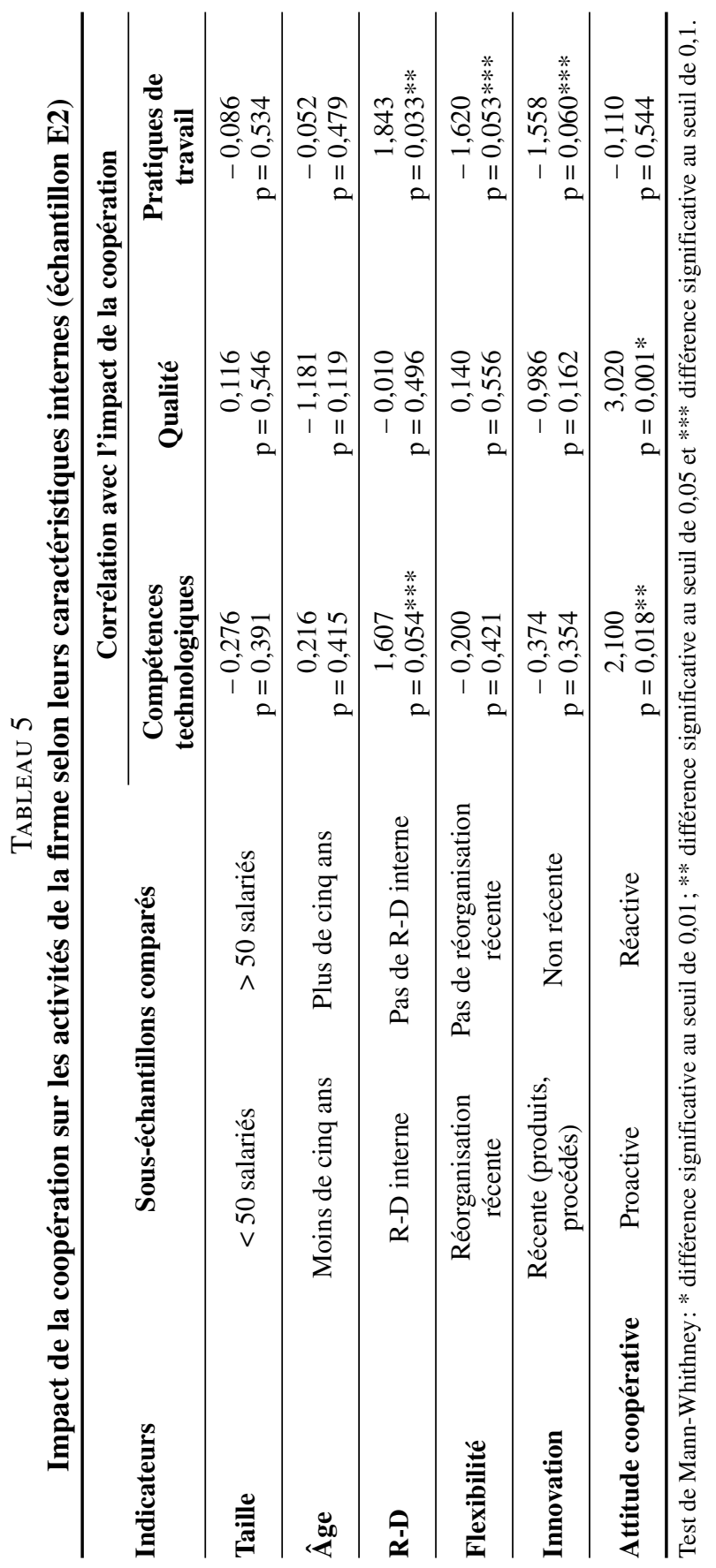

Revue internationale P.M.E., vol. 19, n 1, 2006

(C) 2006 - Presses de l'Université du Québec

Édifice Le Delta I, 2875, boul. Laurier, bureau 450, Québec, Québec G1V 2M2 • Tél.: (418) 657-4399 - www.puq.ca Tiré de: Revue internationale P.M.E., vol. 19, no 1, sous la direction de Louis Raymond • PME1901N Tous droits de reproduction, de traduction et d'adaptation réservés 
Les difficultés structurelles des PME et notamment le manque de ressources disponibles pour l'innovation semblent alors difficilement surmontables à partir d'une seule ouverture sur l'extérieur de ces petites entités. Le dynamisme des PME peut certes être catalysé par le développement de liens interorganisationnels, mais la mise en œuvre des résultats de telles stratégies est fortement conditionnée par les dispositions internes des firmes.

\subsection{Les déterminants externes de l'apprentissage coopératif}

L'étude des caractéristiques de la relation sur l'impact qu'elles peuvent avoir sur l'apprentissage coopératif (voir tableau 6) conduit à trois conclusions, dont deux originales dès lors qu'elles paraissent invalider certaines hypothèses relatives aux déterminants de l'apprentissage: la neutralité de la complémentarité des compétences investies dans la relation sur le développement de nouvelles compétences et le faible impact de la présence initiale d'objectifs d'innovation sur l'intensité des apprentissages réalisés. Le tableau ci-dessous synthétise l'impact des différents indicateurs de la configuration coopérative sur les activités des firmes.

\subsubsection{Les interactions partenariales}

Le premier résultat observé est que les effets d'apprentissage sont d'autant plus prononcés que les interactions entre les partenaires sont importantes. Ainsi, l'échange d'informations sensibles entre les partenaires, jugées comme cruciales dans leurs activités, favorise le développement des capacités d'innovation des partenaires: que ce soit au niveau technologique ou organisationnel, ces échanges augmentent l'impact de la coopération sur les activités des firmes.

La coopération entre une entreprise spécialisée dans le découpage en France et un sous-traitant bulgare spécialisé dans le textile pour se réorienter vers la production de brancards nécessite une communication soutenue: «C'est un petit peu obligé quand même, parce qu'au final, c'est quand même nous qui savons les attentes du client. [...] Ils disent: on a eu un problème sur telle vis, voilà ce qu'on vous propose, ça marchera aussi bien.» La firme française tire ainsi profit des compétences de la seconde: «On est plutôt preneur de ce qu'ils savent, on est utilisateur de leurs idées, de leurs compétences.» Tirer profit des compétences du partenaire passe ainsi par une communication accrue.

De plus, les résultats mettent en évidence que les interactions «sur le terrain», par l'intermédiaire de formation d'équipes communes de travail, renforcent la capacité des partenaires à venir modifier leurs pratiques de travail. Ainsi, au-delà de l'échange d'information, les interactions pratiques facilitent la compréhension et le partage des méthodes de travail réciproques et, donc, l'accès aux connaissances tacites qu'elles incorporent.

Revue internationale P.M.E., vol. 19, $\mathrm{n}^{\circ} 1,2006$

(C) 2006 - Presses de l'Université du Québec

Édifice Le Delta I, 2875, boul. Laurier, bureau 450, Québec, Québec G1V 2M2 • Tél.: (418) 657-4399 - www.puq.ca

Tiré de: Revue internationale P.M.E., vol. 19, no 1, sous la direction de Louis Raymond • PME1901N

Tous droits de reproduction, de traduction et d'adaptation réservés 


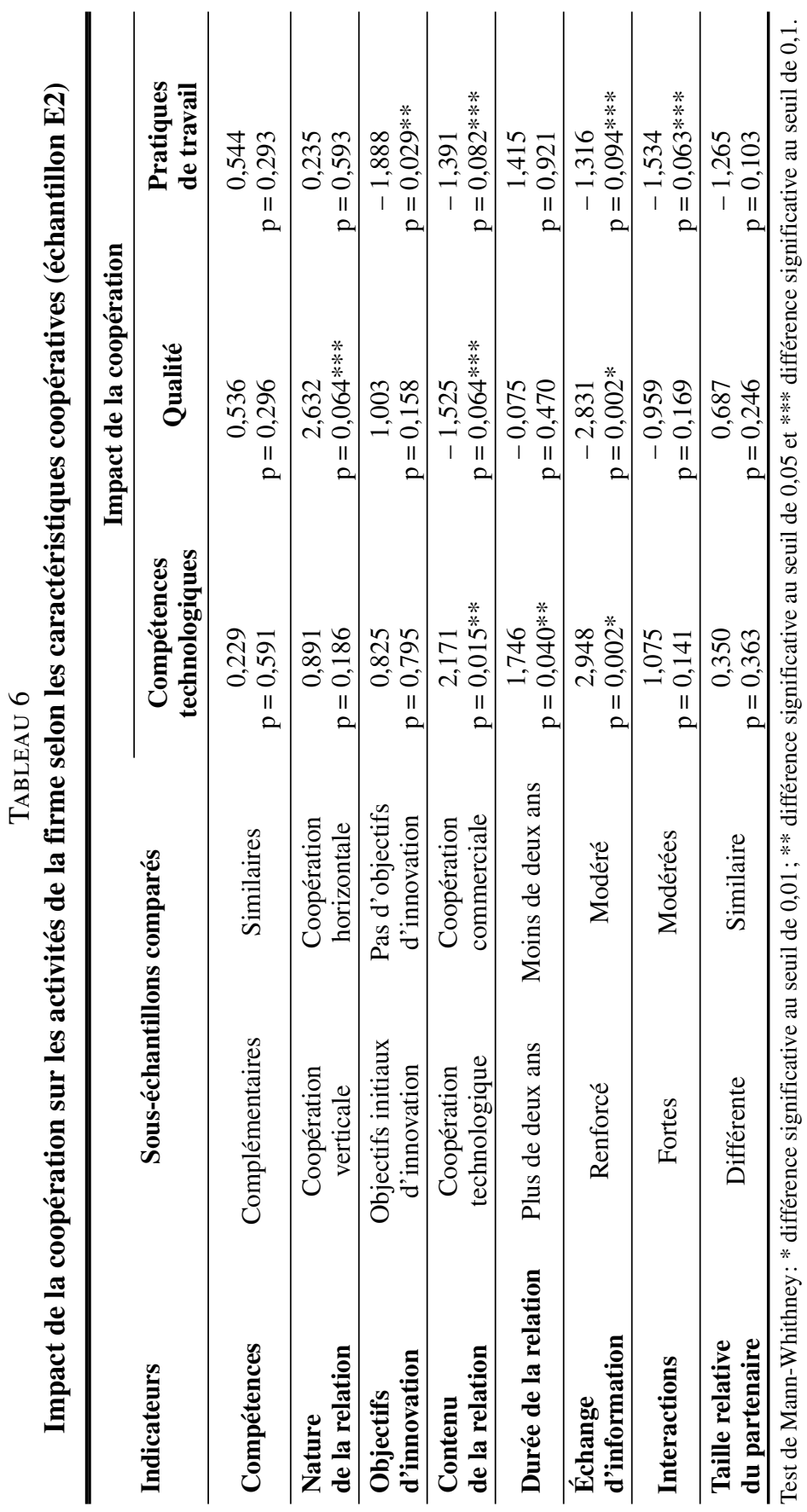

Revue internationale P.M.E., vol. 19, $\mathrm{n}^{\circ}$ 1, 2006

(C) 2006 - Presses de l'Université du Québec

Édifice Le Delta I, 2875, boul. Laurier, bureau 450, Québec, Québec G1V 2M2 • Tél.: (418) 657-4399 - www.puq.ca Tiré de: Revue internationale P.M.E., vol. 19, no 1, sous la direction de Louis Raymond • PME1901N Tous droits de reproduction, de traduction et d'adaptation réservés 
La citation de ce directeur d'entreprise dans la transformation des métaux illustre l'importance de ces interactions partenariales pour faciliter la remise en cause de leurs pratiques de travail. À la suite du travail que certains employés ont effectué chez leur principal client, certaines tâches dans le conditionnement des pièces à fournir ont évolué: «Et là, en revenant, c'étaient les premiers à dire à ceux de l'usine: ah non, faites gaffe, là, il vaut mieux faire comme ça.»

Il est à noter également que l'impact technologique est plus prononcé dans le cadre de relations de plus longue durée. L'apprentissage technologique se développe ainsi sur la durée, à l'inverse de l'apprentissage sur les pratiques de travail, plus immédiat. Cet apprentissage sur les pratiques organisationnelles semble aussi plus prononcé dans les relations verticales au sein d'une filière de production. Il a pu être noté dans les interviews que les partenariats verticaux sont souvent établis par un client afin d'améliorer ces pratiques organisationnelles, notamment pour la mise en place de normes qualité ou de pratiques de travail facilitant la coordination entre les partenaires.

Ces résultats valident l'hypothèse voulant que l'apprentissage coopératif repose sur des interactions fortes entre les partenaires, afin de faciliter les échanges, transferts et croisements de connaissances mutualisées au sein de la relation.

\subsubsection{Les objectifs initiaux d'innovation}

La présence initiale d'objectifs d'innovation pour les partenaires semble n'avoir qu'un impact modéré sur l'apprentissage. En effet, la contribution de cette explicitation initiale de l'innovation comme objectif coopératif n'a d'impact que sur l'amélioration des pratiques de travail. La présence d'objectifs d'innovation n'augmente pas l'impact de la coopération sur les capacités technologiques des partenaires. Cela ne signifie pas que la coopération n'a pas d'impact sur le potentiel technologique des firmes engagées dans des relations partenariales, mais plutôt que, dans le cadre de coopération où ces objectifs ne sont pas affichés, l'impact est comparable.

Cela nous amène à interpréter l'innovation de manière induite par rapport à la coopération. Il apparaît difficile de prévoir ex-ante les retombées d'une relation sur ses propres compétences technologiques (si ce n'est en développant des coopérations technologiques). Le développement de ces compétences apparaît beaucoup plus au fil des opportunités qui se présentent durant le partenariat. L'innovation est alors induite par la coopération beaucoup plus que contrôlée par les partenaires. Cette idée se renforce dès lors que l'on constate que la durée de la relation augmente son impact sur les capacités technologiques. Au fil du temps, des opportunités non envisagées initialement se présentent et qui peuvent se révéler source d'amélioration des compétences technologiques.

Revue internationale P.M.E., vol. 19, $n^{\circ} 1,2006$

(C) 2006 - Presses de l'Université du Québec

Édifice Le Delta I, 2875, boul. Laurier, bureau 450, Québec, Québec G1V 2M2 • Tél.: (418) 657-4399 - www.puq.ca

Tiré de: Revue internationale P.M.E., vol. 19, no 1, sous la direction de Louis Raymond - PME1901N

Tous droits de reproduction, de traduction et d'adaptation réservés 
Un partenariat réunissant deux boyaudiers (l'un français, l'autre marocain) avait pour objectif d'augmenter les capacités de production du producteur français et de préserver un marché en France au second. Au-delà de cet aspect marchand, le producteur français a pu bénéficier des apports technologiques du producteur marocain, notamment le montage des boyaux sur «tube», propre au second: «On avait un tube rigide qui était vendu à l'époque et, moi, j'avais des problèmes. J'ai pu bénéficier de son brevet alors que les autres ne pouvaient pas en profiter.»

\subsubsection{La complémentarité des compétences}

Les résultats présentés n'indiquent aucune différence d'impact sur les capacités d'innovation des firmes entre les coopérations rassemblant des partenaires aux compétences complémentaires et celles rassemblant des compétences similaires. Ce résultat apparaît contre-intuitif par rapport à ce que nous avions pu envisager initialement. En effet, la théorie nous avait amené à poser l'hypothèse que l'apprentissage est souvent stimulé par la mise en relation de base de connaissances différenciées, qui permettent à chaque partenaire de renouveler leurs propres répertoires de connaissances.

Cependant, les entretiens réalisés dans le cadre de l'étude nous ont montré que cette complémentarité était moins considérée comme une source d'apprentissage que comme un rempart contre les risques d'opportunisme inhérents aux relations coopératives. Les compétences juridiques et les capacités des PME à se prémunir contre ce genre de risque restent relativement restreintes (notamment dans le cadre de relations avec des grandes entreprises). L'usage coûteux et complexe des contrats pour les petites entreprises les poussent à développer d'autres garde-fous pour faire face aux aléas relationnels de la coopération. Ainsi, développer des partenariats avec des firmes ne possédant pas les mêmes compétences est souvent considéré comme une première protection contre ces risques (Huet et Lazaric, 2002).

Une étude de cas en Picardie maritime nous a permis d'observer une politique active de développement des groupements d'intérêt économique entre PME, initiée par les institutions publiques locales. C'est le cas de ce GIE à vocation commerciale réunissant plusieurs firmes spécialisées dans le traitement de surface. La complémentarité des compétences entre les partenaires s'est imposée comme une condition nécessaire à la formation de ce groupement, afin d'éviter les problèmes de concurrence entre les partenaires, comme l'illustre cette phrase de l'un des dirigeants de PME: «Si y avait eu concurrence, y aurait jamais eu de groupement. [...] Ah ben c'est clair, moi, si y a un concurrent dans le Zinc, je dirai non. Terminé, y a pas les sept voix pour faire rentrer un membre qui est concurrent. Il peut pas rentrer, c'est clair. C'est très vite fait.»

Revue internationale P.M.E., vol. 19, nº 1, 2006

(C) 2006 - Presses de l'Université du Québec

Édifice Le Delta I, 2875, boul. Laurier, bureau 450, Québec, Québec G1V 2M2 • Tél.: (418) 657-4399 - www.puq.ca

Tiré de: Revue internationale P.M.E., vol. 19, $\mathrm{n}^{\circ} 1$, sous la direction de Louis Raymond • PME1901N

Tous droits de reproduction, de traduction et d'adaptation réservés 
Ou encore, cet autre responsable qui n'envisage pas la possibilité de tirer profit des complémentarités techniques, en raison de leur éloignement trop important de leurs compétences clés: «Quand on fait des visites, on fait un petit tour d'atelier en se disant: c'est joli, c'est rigolo, ça fait du bruit, ça marche bien. Mis à part la curiosité, ça n'a pas d'intérêt pour nous.»

\section{Conclusion}

Les capacités d'innovation des PME sont souvent considérées comme moins importantes en interne en raison des faibles ressources que ces entreprises peuvent consacrer aux stratégies d'apprentissage. Cependant, l'engagement coopératif est censé pouvoir fournir des occasions externes d'apprentissage venant pallier ce déficit interne. L'étude que nous avons présentée révèle néanmoins que ce sont les firmes qui bénéficient de capacités d'innovation en interne qui sont les plus engagées dans des relations coopératives. Ainsi, cette capacité d'absorption et ce dynamisme se traduisent par une meilleure appréhension et mise en pratique des occasions d'apprentissage associées aux stratégies coopératives.

Les résultats de l'apprentissage coopératif sont conditionnés par les caractéristiques internes des entreprises mais également par les caractéristiques de la coopération. Ainsi, la capacité d'absorption (caractéristique organisationnelle interne) facilite l'apprentissage coopératif. Mais les résultats indiquent également que l'intensité des interactions entre les partenaires conditionne l'intensité de ces processus: le partage accru d'informations et l'accès aux pratiques des partenaires augmentent ainsi les opportunités d'apprentissage, ce qui se traduit par un impact plus important sur les capacités d'innovation des entreprises. Si l'innovation peut ainsi apparaître comme un résultat de la coopération, il n’en demeure pas moins que l'identification et l'exploitation de ces occasions reposent fortement sur les capacités d'apprentissage interne des firmes. L'apprentissage coopératif paraît difficile à piloter pour les PME, l'innovation apparaissant de manière induite au fil du partenariat, d'autant plus que les synergies liées à la complémentarité des compétences semblent sous-exploitées (traduisant encore l'importance de la capacité d'absorption pour tirer profit de la coopération). Ces résultats mettent en évidence les limites structurelles (les faibles capacités internes d'apprentissage) et entrepreneuriales pour que les stratégies coopératives puissent servir une dynamique globale d'innovation de ces petites structures.

Les conclusions de notre étude montrent de manière générale l'alternative limitée pour le développement des PME que représentent les coopérations interorganisationnelles: si l'innovation requiert la coopération, cette dernière n'implique pas automatiquement l'innovation. Que ce soit dans leur aptitude à s'engager dans de telles relations ou à tirer profit de ces relations (quand elles existent) pour le renouvellement de leurs connaissances, les PME semblent peu disposées à activer

Revue internationale P.M.E., vol. 19, $\mathrm{n}^{\circ} 1,2006$

(C) 2006 - Presses de l'Université du Québec

Édifice Le Delta I, 2875, boul. Laurier, bureau 450, Québec, Québec G1V 2M2 • Tél.: (418) 657-4399 - www.puq.ca

Tiré de: Revue internationale P.M.E., vol. 19, no 1, sous la direction de Louis Raymond • PME1901N

Tous droits de reproduction, de traduction et d'adaptation réservés 
efficacement le levier coopératif. Dès lors que, de manière autonome, ces firmes restent peu sensibles aux opportunités que peuvent présenter les relations partenariales, des incitations extérieures (par l'intermédiaire de politiques locales de développement, telles que nous avons pu les citer) peuvent faciliter cette diffusion des pratiques coopératives dans le monde des PME. Cependant, pour bénéficier plus intensément de ce développement d'activités collectives, les compétences internes des entreprises doivent également évoluer vers plus de dynamisme entrepreneurial. L'efficacité de telles politiques repose ainsi sur des incitations à la coopération et à l'innovation, qui permettrait de stimuler le développement organisationnel des PME et de dépasser les seuls effets autorenforçants du dynamisme interne et de la coopération.

Ce travail présente à bien des égards un caractère exploratoire et, par conséquent, certaines limites concernant son domaine de validation. En raison du faible taux de coopération, l'échantillon des entreprises déclarant coopérer reste réduit et nécessiterait une extension pour autoriser des tests statistiques affinés. De plus, les résultats présentés ici se situent au plan organisationnel des caractéristiques des coopérations et des PME. Au-delà de ce niveau micro-analytique, des modèles sectoriels de la coopération permettraient d'enrichir et de circonscrire les principales conclusions qui ont pu être explicitées. L'intensité technologique, les contraintes et évolutions environnementales, spécifiques à chaque secteur, permettraient ainsi d'apprécier plus finement les comportements organisationnels et coopératifs. Enfin, à l'issue de ce travail, de nouvelles hypothèses émergent de l'interprétation des résultats statistiques. Les propositions relatives au caractère induit de l'innovation par la coopération et à la gestion des risques d'opportunisme par la complémentarité des compétences nécessiteraient ainsi la construction d'indicateurs pour permettre une testabilité statistique.

\section{Bibliographie}

Alm, H. et M. McKelvey (1999), «When and why does cooperation lead to innovation? An exploration into turbulent waters», Conférence de l'EAPE, Prague.

Audretsch, D.B. (1994) «Small business in industrial economics: the new learning», Revue d'économie industrielle, $\mathrm{n}^{\circ}$ 67, p. 21-38.

Bayona, C., T. Garcia-Marco et E. Huerta (2001), «Firms' motivations for cooperative R-D: an empirical analysis of Spanish firms», Research Policy, n 30, p. 1289-1307.

Belotti, C. (2000), «Les stratégies d'échanges technologiques des petites entreprises industrielles », Revue internationale PME, vol. 13, n 2, p. 75-96.

Bougrain, F. et B. Haudeville (2002), «Innovation, collaboration and SMEs internal research capacities», Research Policy, n 31, p. 735-747.

Cohen, W. et D. Levinthal (1989), «Innovation and learning: the two faces of R-D», The Economic Journal, n ${ }^{\circ}$ 99, p. 569-596.

Revue internationale P.M.E., vol. 19, nº 1, 2006

(C) 2006 - Presses de l'Université du Québec

Édifice Le Delta I, 2875, boul. Laurier, bureau 450, Québec, Québec G1V 2M2 • Tél.: (418) 657-4399 - www.puq.ca

Tiré de: Revue internationale P.M.E., vol. 19, n 1, sous la direction de Louis Raymond • PME1901N

Tous droits de reproduction, de traduction et d'adaptation réservés 
Cohen, W. et D. Levinthal (1990), «Absorptive capacity: a new perspective on learning and innovation», Administrative Science Quarterly, vol. 35, p. 128-152.

COHENDET, P. (1998), «Information, connaissances et théorie évolutionniste de la firme», dans P. Petit, L'économie de l'information: les enseignements des théories économiques, Paris, La Découverte, 405 p.

Fransman, M. (1994), «Information, knowledge, vision and theories of the firm», Industrial and Corporate Change, vol. $3, \mathrm{n}^{\circ} 3$, p. 713-757.

Garette, B. et P. Dussauge (1995), Les stratégies d'alliance, Paris, Les Éditions d'Organisation.

Gulati, R. (1998), «Alliances and networks», Strategic Management Journal, vol. 19, n 4, p. 293-317.

Hannan, M. et J. Freeman (1977), «The population ecology of organisations», American Journal of Sociology, $\mathrm{n}^{\circ}$ 82, p. 929-964.

HelPer, S., J.P. MACDuffie et C. SABEl (2000), «Pragmatic collaborations: advancing knowledge while controlling opportunism», Industrial and Corporate Change, vol. 9 , $\mathrm{n}^{\circ} 3$, p. 443-487.

Hodgson, G. (1988), Economics and Institutions. A Manifesto for a Modern Institutional Economics, Cambridge, Polity Press.

HuEt, F. (2004), Apprentissage collectif et dynamique coopérative: une étude empirique des $P M E$ françaises, Thèse de doctorat, Université de technologie de Compiègne, France.

Huet, F. et N. LAZARIC (2002), «L'émergence de règles et de routines dans les relations inter-firmes: les résultats d'une étude empirique», Colloque institutionnalismes et évolutionnismes, Lyon, France.

Kremp, E. et J. MAIRESSE (2002), «La gestion des connaissances dans l'industrie», Le 4 pages du SESSI, $\mathrm{n}^{\circ} 169$.

LAZARIC, N. et L. MARENGO (2000), «Towards a characterization of knowledge and assets created in technological agreements: some empirical evidence in the automobilerobotics sector», Industrial and Corporate Change, 2000, vol. 9, $\mathrm{n}^{\circ} 2$.

LAzAric, N. et Y. Rizopoulos (dir.) (2003), «Relations inter-firmes: de la création de connaissances complémentaires à la mise en place de nouvelles formes organisationnelles», étude financée par l'État français et la région de Picardie.

LHUILlery, S. (1996), «L'innovation dans l'industrie manufacturière française», dans OCDE, Innovation, brevets et stratégies technologiques.

LuNDVALL, B.A. (1988), «Innovation as an interactive process: from user-producer interaction to the national system of innovation», dans G. Dosi et al. (dir.), Technical Change and Economic Theory, Londres, Pinter, 646 p.

MARCH, J.G. (1991), «Exploration and exploitation in organizational learning», Organisation Science, vol. 2, p. 71-87.

March, J.G. et H.A. Simon (1958), Organizations, Oxford, Blackwell.

Miotti, L. et F. SACHWALD (2003), «Co-operative R-D: why and with whom? An integrated framework of analysis», Research Policy, vol. 32, p. 1481-1499.

Revue internationale P.M.E., vol. 19, nº 1, 2006

(C) 2006 - Presses de l'Université du Québec

Édifice Le Delta I, 2875, boul. Laurier, bureau 450, Québec, Québec G1V 2M2 • Tél.: (418) 657-4399 - www.puq.ca

Tiré de: Revue internationale P.M.E., vol. 19, n 1, sous la direction de Louis Raymond • PME1901N

Tous droits de reproduction, de traduction et d'adaptation réservés 
Munier, F. (2003), «La conjecture schumpétérienne: revue de la littérature empirique et apports de l'économie de la connaissance», Revue d'économie industrielle, $\mathrm{n}^{\circ} 104$, p. 71-84.

Mustar, P. (2001), «Diversité et unité des entreprises à forte croissance du secteur manufacturier en France», Revue internationale PME, vol. 14, n ${ }^{\text {os }} 3-4$, p. 67-89.

Nelson, R. et S. Winter (1982), An Evolutionary Theory of Economic Change, Cambridge, Mass., Harvard University Press.

Noотевоом, B. (1999), Inter-firm Alliances: Design and Analysis, Londres et New York, Routledge.

Nоотевоом, B. (2000), Learning and Innovation in Organizations and Economies, New York, Oxford University Press.

Robertson, P.L. et R.N. Langlois (1994) «Institutions, inertia and changing industrial leadership», Industrial and Corporate Change, vol. 3, n 2, p. 359-378.

Schumacher, E.F. (1978), Small is Beautiful: une société à la mesure de l'homme, Paris, Éditions Contretemps et Seuil. Traduction de Small is Beautiful (1973).

Tether, B.S. (2002), «Who co-operates for innovation, and why. An empirical analysis», Research Policy, vol. 31, p. 947-967.

VoIsin, C. (2000), «Alliances de réseaux et structures de marché: l'émergence d'une regulation pro-compétitive», dans C. Voisin, A. Plunkett et B. Bellon (dir.), La coopération industrielle, Paris, Economica, 320 pages.

Revue internationale P.M.E., vol. 19, n 1, 2006

(C) 2006 - Presses de l'Université du Québec

Édifice Le Delta I, 2875, boul. Laurier, bureau 450, Québec, Québec G1V 2M2 • Tél.: (418) 657-4399 - www.puq.ca

Tiré de: Revue internationale P.M.E., vol. 19, no 1, sous la direction de Louis Raymond • PME1901N

Tous droits de reproduction, de traduction et d'adaptation réservés 